\title{
Low Sidelobe Sparse Concentric Ring Arrays Optimization Using Modified GA
}

\author{
Ke-song Chen, Yong-yun Zhu, Xiao-long Ni, and Hui Chen \\ School of Electronic Engineering, University of Electronic Science and Technology of China, Chengdu 611731, China \\ Correspondence should be addressed to Yong-yun Zhu; zhuyongy1991@sina.cn
}

Received 29 January 2015; Revised 2 April 2015; Accepted 16 April 2015

Academic Editor: Marco Mussetta

Copyright (C) $2015 \mathrm{Ke}$-song Chen et al. This is an open access article distributed under the Creative Commons Attribution License, which permits unrestricted use, distribution, and reproduction in any medium, provided the original work is properly cited.

To minimize the peak sidelobe level (PSLL) of sparse concentric ring arrays, this paper presents an optimization method of grid ring radii of these arrays. The proposed method is based on modified real genetic algorithm (MGA); it makes grid ring radii as optimal variables and makes elements more reasonably distributed on the array aperture. Also, it can improve the PSLL of the sparse concentric ring arrays and can meanwhile control the computational cost. The simulated results confirming the efficiency and the robustness of the algorithm are provided at last.

\section{Introduction}

Ring array can provide ring scan, and simply and flexibly manipulate beam position. It also has ideal directional characteristics in the pitch direction; meanwhile, the symmetry of ring structure makes beam shape, antenna gain, and other properties basically stable and can generally maintain a balance of mutual coupling [1]. Because ring array has these advantages, it is being widely used in many applications, but the PSLL of ring array antenna is relatively higher. Under the conditions that the array aperture and the minimum element spacing are fixed, how to optimize element location to minimize PSLL has therefore become an important research topic $[2,3]$.

Previous studies mostly focused on the thinned arrays that are formed from turning off some elements in a uniform array [4-7]. In [8], genetic algorithm (GA) and particle swarm algorithm are selected to optimize exciting amplitude and phase of thinned concentric array for lower PSLL. In [9], firstly the number of elements was optimized in a thinned concentric array, and then element location was optimized to obtain optimal performance of SLL. Even though elements of sparse concentric ring arrays have greater degrees of freedom and it can make sparse concentric ring arrays get better sidelobe performance, besides [9], there has been little research into the synthesis of sparse concentric ring arrays with nonuniform grid rings so far.
In this paper, the grid ring radii of sparse concentric ring arrays are treated as optimal variables to lower the PSLL. Under the design constraints of the array aperture and the minimum element spacing, the MGA is used to optimize the grid ring radii of the sparse concentric ring arrays. Finally, the good sidelobe performance of sparse antenna arrays has been achieved and the computational complexity of the optimization problem has been effectively controlled.

\section{The Optimal Array Model}

A ring array is a planar array with elements lying on a circle. If several of these arrays with different radii share a common center, then the resulting planar array is a concentric ring array [9].

Array factors for the concentric ring array with a single element at the center (Figure 1) can be described as [9]

$$
\begin{aligned}
F(u, v)= & 1 \\
& +\sum_{n=1}^{H} \omega_{n} \sum_{m=1}^{N_{n}} \exp \left[j k r_{n}\left(\cos \varphi_{m} u+\sin \varphi_{m} v\right)\right],
\end{aligned}
$$

where

$$
\begin{aligned}
& N_{n}=\text { number of elements in ring } n ; \\
& H=\text { number of rings; }
\end{aligned}
$$




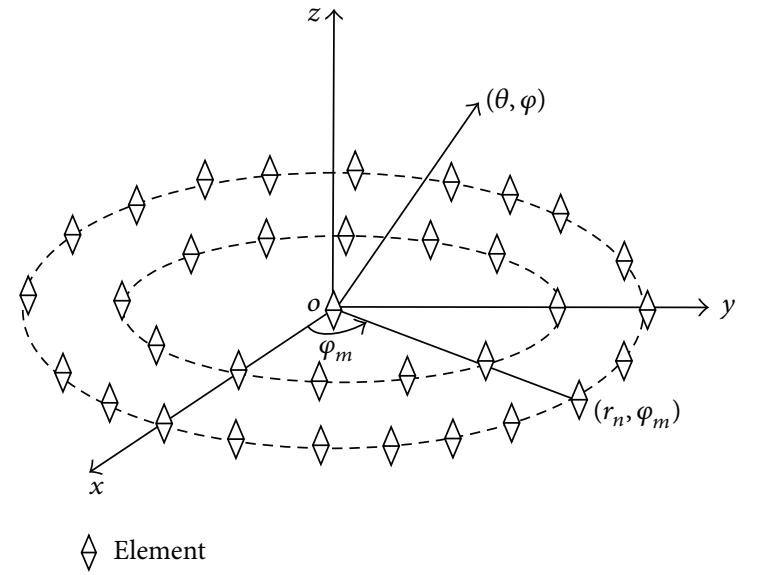

FIGURE 1: Diagram of a concentric ring array.

$$
\begin{aligned}
& k=2 \pi / \lambda ; \\
& \lambda=\text { wavelength; } \\
& \omega_{n}=\text { element weights for ring } n ; \\
& r_{n}=\text { radius for ring } n ; \\
& \left(r_{n} \cos \varphi_{m} u, r_{n} \sin \varphi_{m} v\right)=\text { element location; } \\
& u=\sin \theta \cos \varphi ; \\
& v=\sin \theta \sin \varphi ; \\
& \varphi_{m}=2 \pi(m-1) / N_{n} .
\end{aligned}
$$

Elements are ideal omnidirectional antenna units, each element has uniform amplitude and phase excitation, and direction of main beam of array points toward normal direction of the array. When elements of sparse concentric ring antenna arrays are distributed evenly on grid rings, and the grid ring spacing is nonuniform, the resulting concentric ring array is a sparse concentric ring array [10]. Assuming an element is fixed at the common center of grid rings, we can write array factors for the sparse concentric ring array as

$$
F(u, v)=1+\sum_{i=1}^{N-1} \omega_{i} \exp \left[j k\left(r_{n} \cos \varphi_{i} u+r_{n} \sin \varphi_{i} v\right)\right]
$$

where $N$ is the number of elements.

When the sparse concentric ring array here has $H$ grid rings with radii $r_{0}, r_{1}, \ldots, r_{H}$ and has optimization constraints including the array aperture and the minimum element spacing, we treat the radii of grid rings as optimal variables; the optimization goal is to minimize PSLL of the sparse concentric ring array, and thus the optimization is described as

$$
\begin{gathered}
\operatorname{Min}\left\{\operatorname{PSLL}\left(r_{0}, r_{1}, r_{2}, \ldots, r_{H}\right)\right\} \\
N_{n}=\left\lfloor\frac{2 \pi r_{n}}{d_{n}}\right\rfloor, \quad 0<d_{c} \leq d_{n}<\lambda, n=1,2, \ldots, H, \\
\sum_{n=0}^{H} N_{n}=N, \\
\sum_{n=1}^{H} \frac{2 \pi r_{n}}{\lambda}+1<N \leq \sum_{n=1}^{H} \frac{2 \pi r_{n}}{d_{c}}+1,
\end{gathered}
$$

where

$$
\operatorname{PSLL}\left(r_{0}, r_{1}, r_{2}, \ldots, r_{H}\right)=\max \left\{\left|\frac{F(u, v)}{F_{\text {max }}}\right|\right\},
$$

where $N_{n}$ is the number of elements in ring $n, d_{n}$ is element spacing in ring $n, d_{c}$ is the design constraint of minimum element spacing, and $\mathrm{FF}_{\max }$ is the peak of main beam; the region of $u$ and $v$ for which fitness is valid excluding the main beam. When element spacing of each ring is equal to $d_{c}$, the number of elements $N$ obtains maximum value $\sum_{n=1}^{H} 2 \pi r_{n} / d_{c}+1$; when element spacing of each ring is equal to $\lambda$, the number of elements $N$ obtains minimum value $\sum_{n=1}^{H} 2 \pi r_{n} / \lambda+1$. Since the number of elements must be an integer, the value of $N_{n}$ must be rounded up or down. To keep $d_{n} \geq d_{c}$ and allow sufficient element spacing, the digits to the right of the decimal point are discarded.

\section{Optimization of Grid Ring Radii Using MGA}

In [11], MGA was proposed to indirectly optimize variables for sparse linear array antenna. In this communication MGA similarly avoids directly describing the optimization variables, that is, the grid ring radii. This technology way can be used to effectively reduce the search space. The main steps of MGA for sidelobe suppression of sparse concentric ring arrays by optimizing the grid ring radii are as follows.

3.1. Set Up the Initial Population. Let the optimal variable $\mathbf{R}_{i}=\left[r_{0}, r_{1}, \ldots, r_{H}\right]$ act as the individual; it is a real vector. In order to meet the design constraint of minimum element spacing, thus

$$
r_{n}=r_{n-1}+d_{c}+\Delta_{n}
$$

where $r_{0}=0,0<\Delta_{n}<\lambda / 2,1 \leq n \leq H$, and $r_{H}=0.5 L$, $L /\left(2 d_{c}+\lambda\right)<H<L / 2 d_{c}$, where $L$ is the design constraint of the array aperture.

In this paper, we assign the population which contains $S$ individuals a letter $\mathbf{R}$.

3.2. Crossover and Mutation. If the $i$ th and $j$ th individuals of population are selected, and single-point crossover is 
TABLE 1: Hybrid genetic algorithm (HGA), peak sidelobe level (PSLL), modified real genetic algorithm (MGA), number of elements (N), run time (RT), ring radii $\left(r_{n}\right)$, and number of elements in the rings $\left(N_{n}\right)$.

\begin{tabular}{|c|c|c|c|c|c|c|c|c|c|c|c|c|}
\hline Method & PSLL (dB) & RT & $N$ & $n$ & 1 & 2 & 3 & 4 & 5 & 6 & 7 & 8 \\
\hline \multirow{2}{*}{ HGA [9] } & \multirow{2}{*}{-22.94} & \multirow{2}{*}{$3 \mathrm{~h}$} & \multirow{2}{*}{201} & $r_{n}(\lambda)$ & 1.00 & 1.59 & 2.14 & 2.88 & 3.66 & 4.98 & - & - \\
\hline & & & & $N_{n}$ & 12 & 19 & 26 & 36 & 45 & 62 & - & - \\
\hline \multirow{2}{*}{ The best result Opt. $r_{n}$ by MGA } & \multirow{2}{*}{-29.13} & \multirow{2}{*}{$1.7 \mathrm{~h}$} & \multirow{2}{*}{201} & $r_{n}(\lambda)$ & 0.5590 & 1.0642 & 1.6062 & 2.1092 & 2.6404 & 3.3381 & 4.0530 & 4.9800 \\
\hline & & & & $N_{n}$ & 7 & 13 & 20 & 24 & 27 & 32 & 36 & 41 \\
\hline \multirow{2}{*}{ The worst result Opt. $r_{n}$ by MGA } & \multirow{2}{*}{-27.93} & \multirow{2}{*}{$1.7 \mathrm{~h}$} & \multirow{2}{*}{201} & $r_{n}(\lambda)$ & 0.5450 & 1.0460 & 1.5511 & 2.1021 & 2.6776 & 3.3155 & 4.0063 & 4.9800 \\
\hline & & & & $N_{n}$ & 6 & 12 & 19 & 25 & 29 & 33 & 38 & 38 \\
\hline
\end{tabular}

produced at the $n$th gene, then the two new individuals after crossover can be expressed as

$$
\begin{aligned}
\mathbf{R}_{i} & =\operatorname{sort}\left[0, R_{1 i}, R_{2 i}, \ldots, R_{n j}, \ldots R_{H i}\right] \\
\mathbf{R}_{j} & =\operatorname{sort}\left[0, R_{1 j}, R_{2 j}, \ldots, R_{n i}, \ldots R_{H j}\right],
\end{aligned}
$$

where

$$
\begin{array}{r}
d_{c} \leq\left|R_{n i}-R_{m j}\right|<\lambda, \\
d_{c} \leq\left|R_{n i}-R_{m j}\right| \leq \lambda, \\
m=1,2, \ldots, H, m \neq n \\
d_{c} \leq\left|R_{n j}-R_{m i}\right|<\lambda, \\
d_{c} \leq\left|R_{n j}-R_{m i}\right| \leq \lambda, \\
m=1,2, \ldots, H, m \neq n .
\end{array}
$$

If the $z$ th gene of the $l$ th individual is mutation, the new individual after mutation can be expressed as

$$
\mathbf{R}_{l}^{\prime}=\operatorname{sort}\left[0, R_{1 l}, R_{2 l}, \ldots, R_{(z-1) l}, \xi, R_{(z+1) l}, \ldots, R_{H l}\right],
$$

where $\xi \subseteq(0,0.5 L)$, and $d_{c} \leq\left|\xi-R_{i l}\right|<\lambda, 0 \leq i \leq H$ and $i \neq z$. The number of genes crossover and mutation is determined by the given crossover and mutation rate.

3.3. Fitness Calculation. Filial generation population can be obtained after selection, crossover, and mutation; namely,

$$
\begin{aligned}
& \mathbf{R}^{\prime}=\left[\mathbf{R}_{1}^{\prime}, \mathbf{R}_{2}^{\prime}, \ldots, \mathbf{R}_{s}^{\prime}, \ldots, \mathbf{R}_{S}^{\prime}\right] \\
& \mathbf{R}_{s}^{\prime}=\left[0, R_{s 1}, R_{s 2}, \ldots, R_{s n} \ldots, R_{s H}\right] .
\end{aligned}
$$

As radii and $d_{n}$ are known, $N_{n}$ can be expressed as

$$
N_{n}=\left\lfloor\frac{2 \pi R_{s n}}{d_{n}}\right\rfloor .
$$

According to $\varphi_{m}=2 \pi(m-1) / N_{n}, \varphi_{m}$ can be calculated. Thus the element location $\left(r_{n} \cos \varphi_{m} u, r_{n} \sin \varphi_{m} v\right)$ can be obtained.

The array factors can be calculated by substituting the element location and radii into (2), and (4) can evaluate the fitness of the new individual. If iteration process meets the maximum number of generations, the MGA will end.

The essential steps of the modified GA are summarized in [11].

\section{Simulation Results}

In order to illustrate the effectiveness of this optimization method, this paper gives two examples to compare with sparse arrays obtained in [9]. For this sparse concentric ring array synthesis, basic parameters of MGA are set as follows: population includes 100 individuals; truncation selection (discard bottom 50\%); the crossover and mutational rate is 0.2 and 0.02 , respectively; the number of generations is 200 . Elitism is also employed.

4.1. Sparse Concentric Ring Arrays with 8 Grid Rings. According to [9], let number of elements $N=201$, minimum element spacing $d_{c}=0.5 \lambda$, and the array aperture $L=$ 9.96 $\lambda$. Convergence characteristics are averaged from 5 independent runs. It takes about $1 \mathrm{~h} 44 \mathrm{~min}$ to complete a single trial. Figure 2(a) shows the average, the best, and the worst progress of the radii optimization as the function of the number of generation. Figure 2(b) shows the radiation pattern of the best sparse concentric ring array. The element configuration of the best sparse concentric ring array is shown in Figure 2(c). The best result and the worst result are presented in Table 1 to contrast with the best result based on hybrid genetic algorithm (HGA) in [9]. Using the same objective function and the number of elements, the best optimization result by the optimization method of grid ring radii is $6.19 \mathrm{~dB}$ lower than that of the best result based on HGA in [9]. The simulation results verify that the optimization method of grid ring radii has preferable efficiency, and as shown in Figure 2(a), the optimization method of grid ring radii has good robustness and convergence characteristics.

4.2. Sparse Concentric Ring Arrays with 6 Grid Rings. According to [9], let number of elements $N=142$, minimum element spacing $d_{c}=0.5 \lambda$, and the array aperture $L=9.4 \lambda$. Convergence characteristics are averaged from 5 independent runs. It takes about $1 \mathrm{~h} 10 \mathrm{~min}$ to complete a single trial. Figure 2(d) shows the average, the best, and the worst progress of the grid radii optimization as the function of the number of generation. Figure $2(\mathrm{e})$ shows the radiation pattern of the best result. The element configuration of the best sparse concentric ring array is shown in Figure 2(f). The best result and the worst result are presented in Table 2 to contrast with the best result by optimizing $r_{n}$ and $N_{n}$ in [9]. Using the same objective function, the number of elements and the number of rings, the optimization result by 


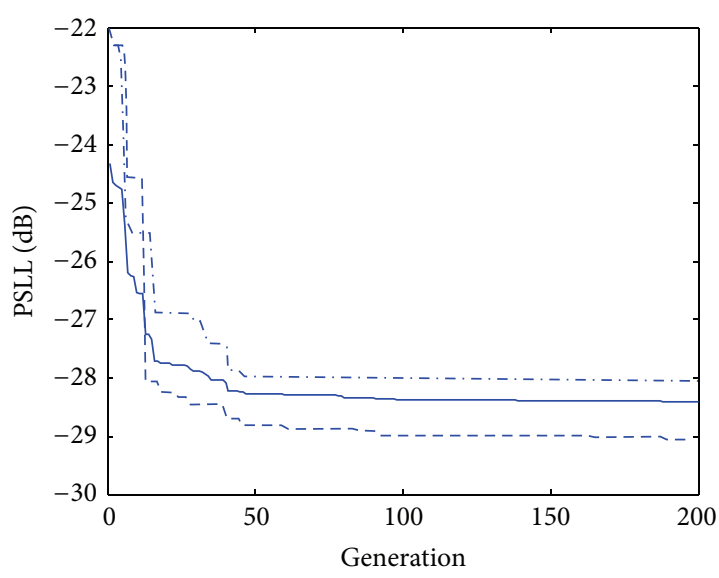

- Convergent curve of 5 trials averaged . - - Convergent curve of the worst single trial Convergent curve of the best single trial

(a) Convergence characteristics

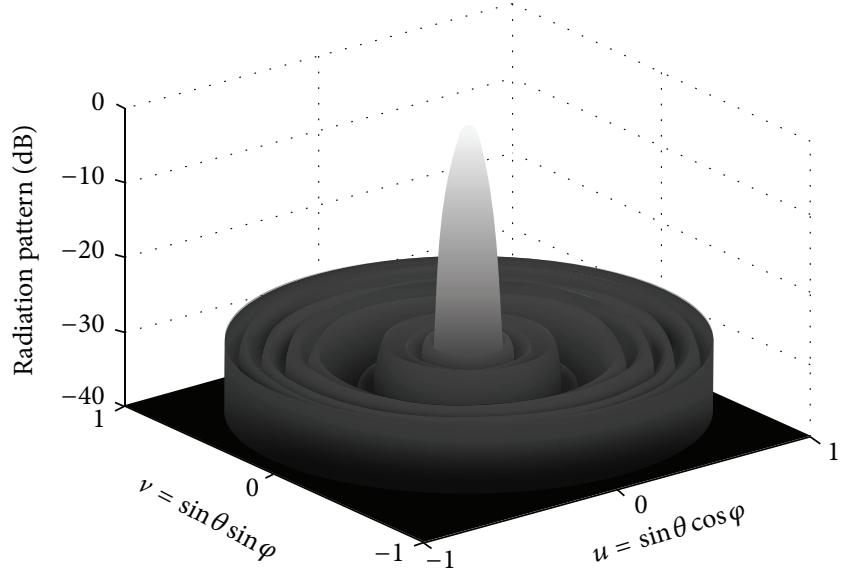

(b) Radiation pattern of the best result

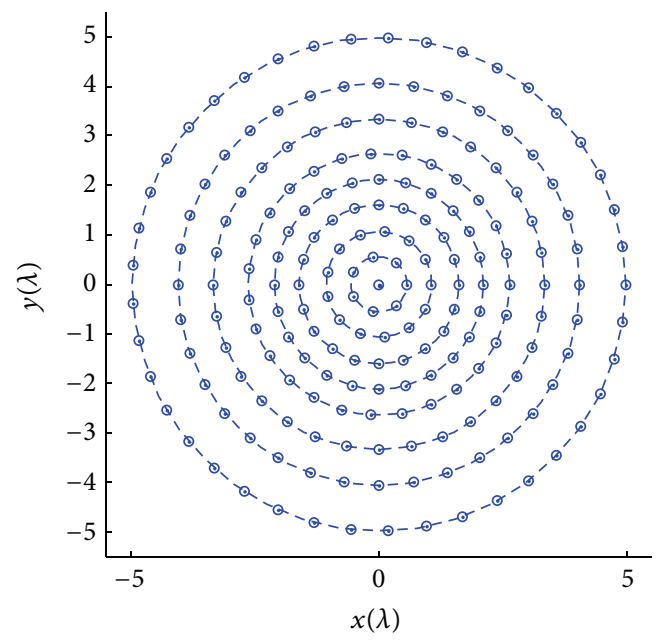

(c) The element configuration of the best sparse

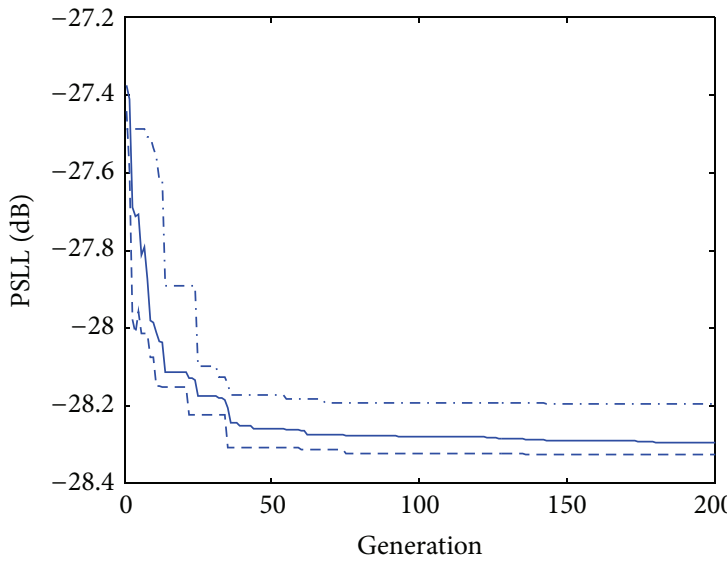

- Convergent curve of 5 trials averaged

. . . - Convergent curve of the worst single trial

- - - Convergent curve of the best single trial

(d) Convergence characteristics in simulation 4.2

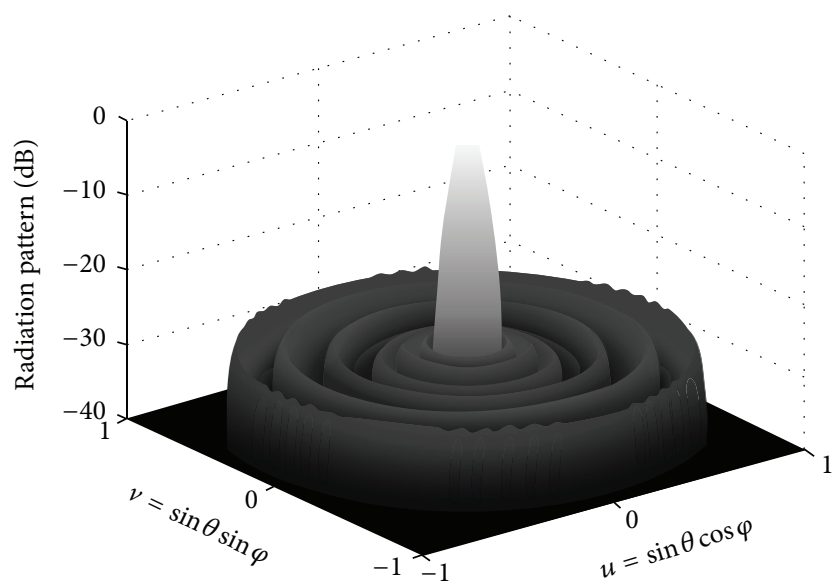

(e) Radiation pattern of the best result in simulation 4.2

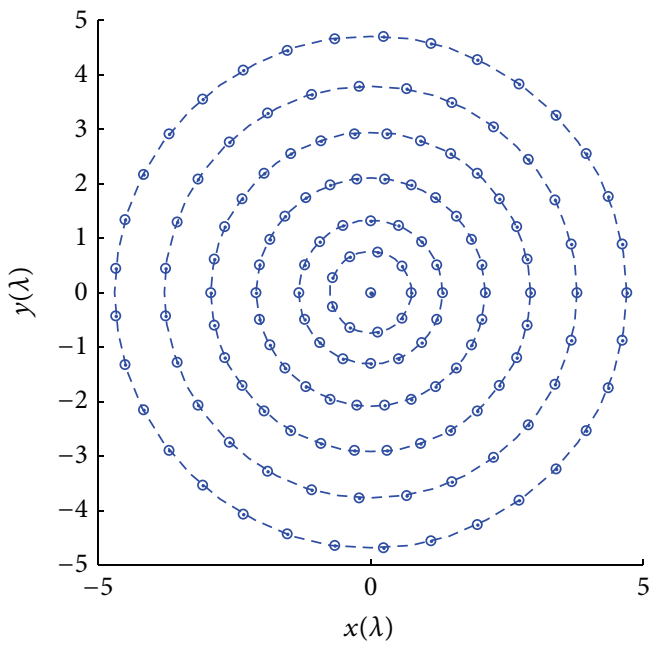

(f) The element configuration of the best sparse

FIGURE 2: Convergence characteristics, element configuration, and radiation pattern for the sparse concentric ring arrays. 
TABLE 2: Peak sidelobe level (PSLL), modified real genetic algorithm (MGA), number of elements $(N)$, run time (RT), ring radii $\left(r_{n}\right)$, and number of elements in the rings $\left(N_{n}\right)$.

\begin{tabular}{lcccccccccc}
\hline Method & PSLL $(\mathrm{dB})$ & $\mathrm{RT}$ & $N$ & $n$ & 1 & 2 & 3 & 4 & 5 \\
\hline Opt. $r_{n} \& N_{n}[9]$ & \multirow{2}{*}{-27.82} & \multirow{2}{*}{$57 \mathrm{~min}$} & \multirow{2}{*}{142} & $r_{n}(\lambda)$ & 0.76 & 1.36 & 2.09 & 2.99 & 3.78 & 4.70 \\
& & & & $N_{n}$ & 9 & 17 & 25 & 31 & 26 & 33 \\
The best result Opt. $r_{n}$ by MGA & -28.33 & \multirow{2}{*}{$70 \mathrm{~min}$} & \multirow{2}{*}{142} & $r_{n}(\lambda)$ & 0.7467 & 1.3180 & 2.0969 & 2.9305 & 3.7852 & 4.7000 \\
& & & & $N_{n}$ & 9 & 16 & 26 & 30 & 27 & 33 \\
The worst result Opt. $r_{n}$ by MGA & -28.18 & \multirow{2}{*}{$70 \mathrm{~min}$} & \multirow{2}{*}{142} & $r_{n}(\lambda)$ & 0.7506 & 1.3173 & 2.0726 & 2.9078 & 3.7691 & 4.7000 \\
& & & & $N_{n}$ & 9 & 17 & 25 & 30 & 27 & 33 \\
\hline
\end{tabular}

the optimization method of grid ring radii is $0.51 \mathrm{~dB}$ lower than that of the best result in [9].

Both the simulations in this paper are completed in MATLAB2013 on a PC (Intel (R) Core (TM) i5-4570 processor $3.2 \mathrm{GHz}$ ). The following numerical results demonstrate that the optimization method of grid ring radii runs well with great efficiency and considerable stability. Compared with the synthesis technique described in [9], the proposed method of grid ring radii based on MGA reaches the same goal through different routes.

\section{Conclusion}

An optimization method of grid ring radii of sparse concentric ring arrays based on MGA is presented in this paper. Compared with [9], although the synthesis method presented in this paper is iterative techniques in nature, it can cope with the design constraints of the array aperture and the minimum element spacing, and it uses grid ring radii as optimal variables to make elements more reasonably distributed on the array aperture. Hence, it may be more practical under some special circumstances. This method not only provides useful ways for solving optimization problems of such arrays, but also provides a valuable reference for practical engineering.

\section{Conflict of Interests}

The authors declare that there is no conflict of interests regarding the publication of this paper.

\section{Acknowledgment}

This work was supported by the Natural Science Foundation of China (no. U1233103).

\section{References}

[1] U. Singh and T. S. Kamal, "Synthesis of thinned planar concentric circular antenna arrays using biogeography-based optimisation," IET Microwaves, Antennas \& Propagation, vol. 6, no. 7, pp. 822-829, 2012.

[2] B. P. Kumar and G. R. Branner, "Generalized analytical technique for the synthesis of unequally spaced arrays with linear, planar, cylindrical or spherical geometry," IEEE Transactions on Antennas and Propagation, vol. 53, no. 2, pp. 621-634, 2005.
[3] W. Zhang, L. Li, and F. Li, "Reducing the number of elements in linear and planar antenna arrays with sparseness constrained optimization," IEEE Transactions on Antennas and Propagation, vol. 59, no. 8, pp. 3106-3111, 2011.

[4] D. Mandal, A. Majumdar, R. Kar, and S. P. Ghoshal, “Thinned concentric circular array antennas synthesis using genetic algorithm," in Proceedings of the IEEE Student Conference on Research and Development (SCOReD '11), pp. 194-198, Putrajaya, Malaysia, December 2011.

[5] D. Mandal, R. Kar, and S. P. Ghoshal, "Thinned concentric circular array antenna synthesis using particle swarm optimization with constriction factor and inertia weight approach," in Proceedings of the International Conference on Recent Trends in Information Systems (ReTIS '11), pp. 77-81, Kolkata, India, December 2011.

[6] P. Ghosh and S. Das, "Synthesis of thinned planar concentric circular antenna arrays-a differential evolutionary approach," Journal of the Progress in Electromagnetics Research B, vol. 29, pp. 63-82, 2011.

[7] D. Mandal, A. Majumdar, R. Kar, and S. P. Ghoshal, "Thinned concentric circular array antennas synthesis using genetic algorithm," in Proceedings of the IEEE Student Conference on Research and Development (SCOReD '11), pp. 194-198, IEEE, Cyberjaya, Malaysia, December 2011.

[8] A. Reyna and M. A. Panduro, "Design of steerable concentric rings array using rotation properties and evolutionary optimization," in Proceedings of the 4th European Conference on Antennas and Propagation (EuCAP '10), pp. 1-5, IEEE, Barcelona, Spain, April 2010.

[9] R. L. Haupt, "Optimized element spacing for low sidelobe concentric ring arrays," IEEE Transactions on Antennas and Propagation, vol. 56, no. 1, pp. 266-268, 2008.

[10] S. Xuejun, G. Quan, and T. Bin, "Design of sparse concentric multi-circular array using micro-genetic optimum algorithm," in Proceedings of the IEEE Communications and Mobile Computing, pp. 51-55, Yunnan, China, January 2009.

[11] K. Chen, Z. He, and C. Han, "A modified real GA for the sparse linear array synthesis with multiple constraints," IEEE Transactions on Antennas and Propagation, vol. 54, no. 7, pp. 2169-2173, 2006. 

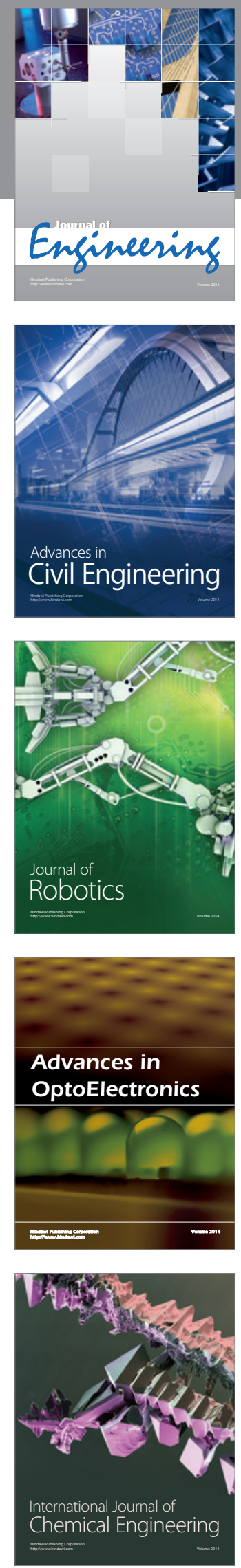

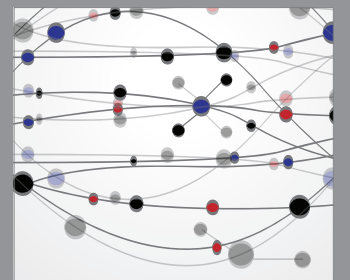

The Scientific World Journal
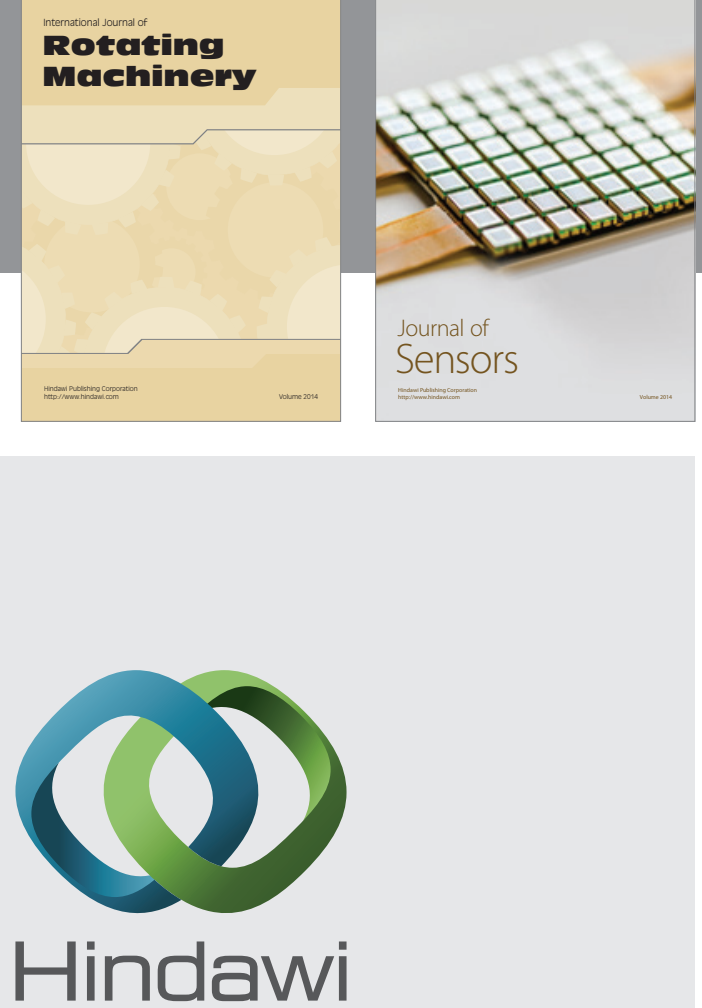

Submit your manuscripts at http://www.hindawi.com
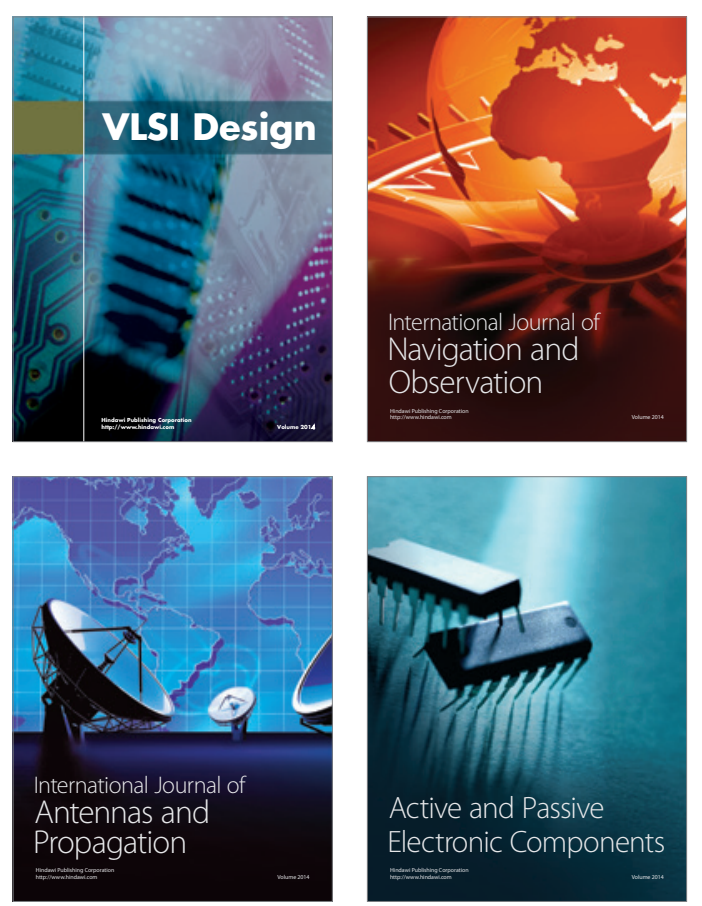
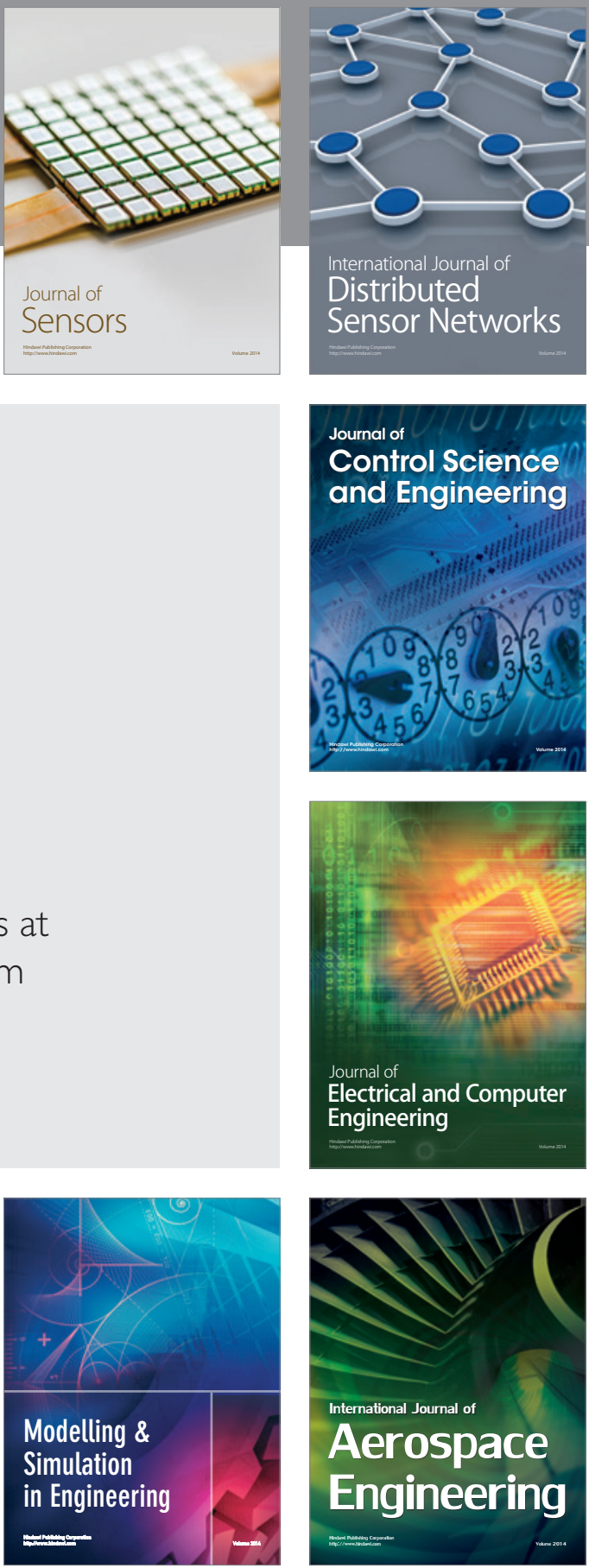

Journal of

Control Science

and Engineering
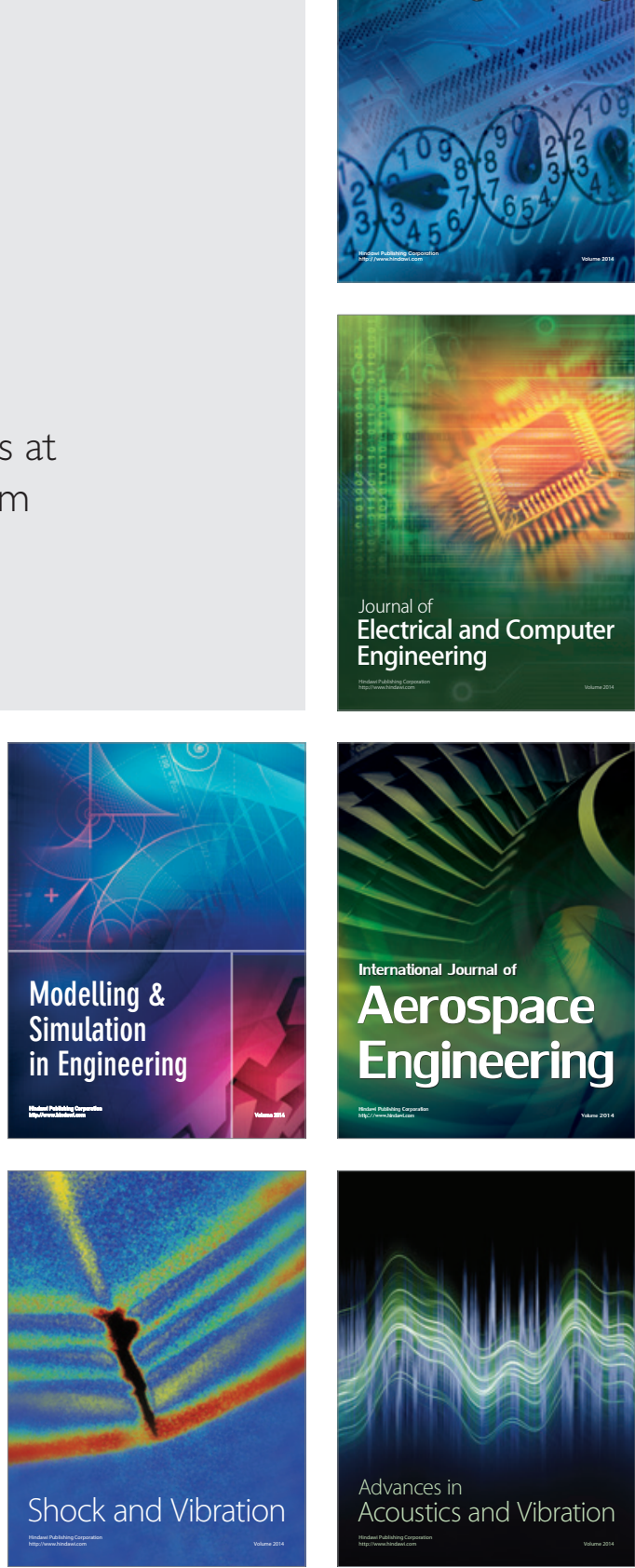